\title{
Fabrication of PLGA/ (HA/CS) n/CD34 Bioactive Multiplayer Film on
}

\section{Stainless Steel Stent}

\author{
Ying $\mathrm{Li}^{1}$, Hong Zhao ${ }^{2}$, Shixuan Zhang ${ }^{2}$ \\ ( ${ }^{1}$ Liaoning Geology Engineering Vocation College, \\ ${ }^{2}$ Dalian University of Technology)
}

\begin{abstract}
At present PLGA is widely used in clinical as a drug carrier in drug-eluting stent coating. But due to the existence of poor biological activity and easily cased thrombosis of PLGA, it is serious to increase the biological activity and hemocompatibility of drug-eluting stent. In this research, the paper try to fabricate PLGA/ (HA/CS) n/CD34 bioactive multilayer films on biomedical 316L stainless steel (316LSS) surface via electrostatic self-assembly. Using fluorescent dyes to make the characterization of the films. It is considered the effect of corrosion resistance in blood about solution concentration and layers of multilayer films through blood washed simulation experiments. The research makes platelet adhesion experiment on multilayer films in different solution concentration and layers in order to optimize process parameters.
\end{abstract}

KEYWORDS: Electrostatic self-assembly; multilayer films; CD34 antibody; Polylactide-co-glycolide (PLGA)

\section{Introduction}

The restenosis after percutaneous transluminal angioplasty (PTA) will seriously affect its long-term effect. Endovascular stenting is possible to reduce the restenosis rate, but restenosis is still the main reason that disturbs the long-term efficacy of interventional therapy. According to statistics, the incidence of restenosis within 1 year can reach $20 \%$. In recent years, some researchers are committed to the pathway of accelerating damage endometrial repair, to achieve this objective, which is called cell coated stent. Especially CD34 antibody stent is able to capture endothelial progenitor cells (EPC), and accelerate the endothelial repair, so it has a broad prospect in clinical application. The endothelial progenitor cells flow in blood vessels with the blood, and after intimal is damaged, they can be gathered at the site of injury, and quickly repair the damaged endothelial and prevent thrombosis. The surface of EPC can express CD34 antigen relatively specifically, so according to the antigen antibody binding principle, using Stent to cover CD34 antibody until EPC is attached to the stent, can accelerated the damaged endothelium repair, and then achieve lower restenosis and prevent stent thrombosis. The purpose of this paper is to construct the CD34 antibodies multilayers on stainless steel stent, so as to verify the presence of the multilayer film and test its erosion performance and biocompatibility.

\section{Experiment}

\section{A. Experimental Materials}

Respectively with acetone, ethanol and ultra-pure water, do ultrasonic cleaning of the stainless steel stent for 20 minutes, and dry it for preparation. Compound: 
1) $4 \%$ PLGA solution (PLA: PGA=7:3, $\mathrm{M}_{\mathrm{W}}$ $=150$ thousand, solvent of chloroform);

2) PEI solution (PEI: branching, molecular weight 70000 , concentration $5 \mathrm{mg} / \mathrm{ml}$, solvent of $0.9 \%$ sodium chloride solution, $\mathrm{PH}=7.40$ );

3) Chitosan solution (CS: deacetylation degree > 85\%, molecular weight 200000 , concentration $1 \mathrm{mg} / \mathrm{ml}, 2 \mathrm{mg} / \mathrm{ml}, 5 \mathrm{mg} / \mathrm{ml}$, solvent of acetic acid solution, $\mathrm{PH}=4.00$ );

4) Hyaluronic acid solution (HA: molecular weight 600000 , concentration $1 \mathrm{mg} / \mathrm{ml}, 2$ $\mathrm{mg} / \mathrm{ml}, 5 \mathrm{mg} / \mathrm{ml}$, solvent of deionized water, $\mathrm{PH}=6.00$ );

5) Buffer solution (potassium dihydrogen phosphate $1.2 \mathrm{~g}$, dipotassium hydrogen phosphate $0.2 \mathrm{~g}$, sodium chloride $1.5 \mathrm{~g}$, dissolved in $1000 \mathrm{ml}$ deionized water, $\mathrm{PH}$ adjusted as 7.40 , $6.00,4.00)$;

6) Antibody binding agent (provided by Zhang Shixuan from chemical college of Dalian University of Technology);

7) Mouse anti human primitive hematopoietic cells (CD34) monoclonal antibody working solution;

8) Rhodamine-labeled goat anti-mouse $\operatorname{IgG}$ antibody solution.

\section{B. Fabrication of Self-assembled Multilayers}

Immerse the stent into PLGA solution and then pull it out, and place it in ultra-clean chamber for 12 hours, until it forms film. Place the PLGA coating into 50\% ethanol for ultrasonic vibration for 20 minutes, and washed with deionized water drying once taking out. Immerse it into OEI solution, standing for 3.5 hours of surface cationic. Then take out and wash with $\mathrm{PH}=7.40$ buffer solution and dry it, prepared for self-assembly of biomolecules polyelectrolyte next step.

Immerse the PLGA film after PEI activation into hyaluronic acid solution for 20 minutes of standing. Then take it out, and wash with
$\mathrm{PH}=6.00$ buffer solution, and immerse it into Chitosan solution again for 20 minutes. Finally take it out, and wash with $\mathrm{PH}=4.00$ buffer solution, forming one electrostatic self-assembled multilayers. Repeatedly immerse and still it, until it forms multilayer films.

(1) Characterization of Self-assembled Multilayers

With Olympus BX51 fluorescence microscopy, observe the fluorescence microscopy picture of antibody coating and Rhodamine-labeled goat anti-mouse $\operatorname{IgG}$ antibody combined; conduct simulated body fluid scouring experiment of PLGA/(HA/CS)n/CD34 stent.

(2) Platelet Adhesion Experiment

After centrifugal of the fresh ant-coagulated blood for 10 minutes, then take out the upper part of the platelet rich plasma. Immerse the sample into $37^{\circ} \mathrm{C}$ platelet solution for 1 hour, and then take out. Then rinse it with saline solution, and fix it with $2.5 \%$ glutaraldehyde fixative solution. Then dehydrate with alcohol series and dealcoholize with isoamyl acetate. After drying at critical point, spray silver, and observe platelet morphology with biological microscope and scanning electron microscope.

\section{Results and Discussion}

\section{A. Erosion Simulation Experiment of Coated Stents in Vitro}

In this experiment, firstly we use peristaltic pump to adjust the speed of the simulated body fluid circulation until close to the speed of the human arterial blood circulation $(0.5 \mathrm{~m} / \mathrm{s})$, and then place the sample into the cycle path for erosion test. To better simulate the environment of human blood, place the simulated body fluid in $37{ }^{\circ} \mathrm{C}$ constant temperature water bath box. Respectively after $24 \mathrm{~h}, 48 \mathrm{~h}$ and $72 \mathrm{~h}$, take out 
the sample, and then observe the degree of coating desorption after staining. The simulated body fluid is Kokubo, because the specie the content of it containing ions is similar to that of arterial blood, thus could well simulate human blood environment.

Table 1. Component of Kokubo stimulate body liquid ( $\mathrm{m} \mathrm{mol}$ )

\begin{tabular}{|l|l|l|l|l|l|l|l|}
\hline $\mathrm{Na}^{+}$ & $\mathrm{HCO}_{3}{ }^{-}$ & $\mathrm{K}^{+}$ & $\mathrm{HPO}_{4}{ }^{2-}$ & $\mathrm{Mg}^{+}$ & $\mathrm{Ca}^{+}$ & $\mathrm{SO}_{4}{ }^{2-}$ & $\mathrm{Cl}^{-}$ \\
\hline 142.0 & 27.0 & 5.0 & 1.0 & 1.5 & 2.5 & 0.5 & 147.8 \\
\hline
\end{tabular}

As shown in Figure 1, A, B, C, D are fluorescent tag photos that before and after erosion test of different periods. According to the fluorescence, we can measure the residual amount of coating antibody.

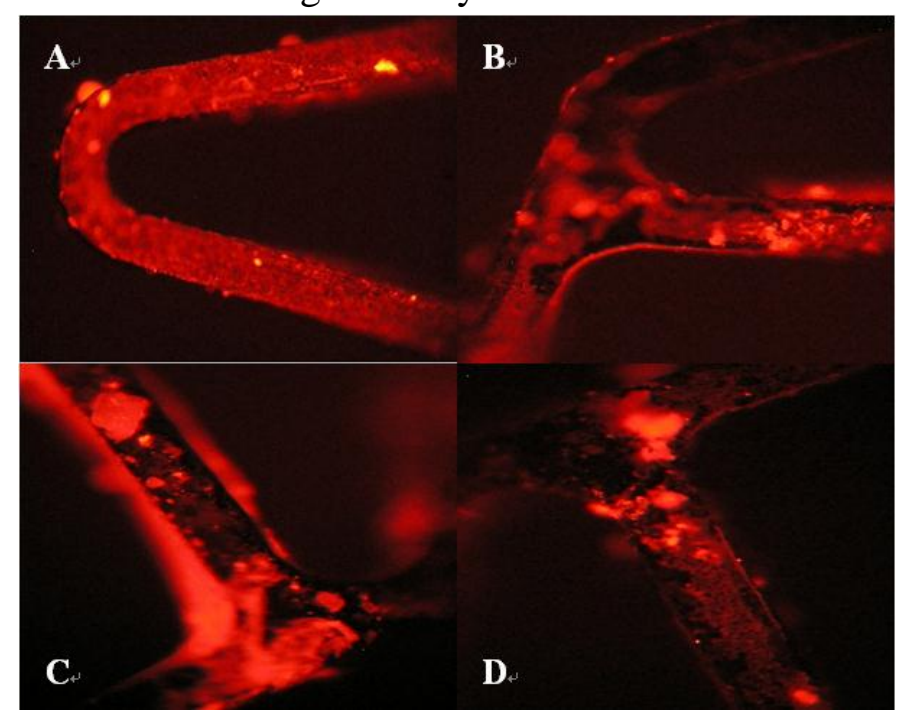

Figure 1. Fluorescent images of

PLGA/(CS/HA)4/CD34/HA coating with blood washed simulation experiments(Concentration of HA and CS is $2 \mathrm{mg} / \mathrm{ml})$

A. fluorescent images before experiment; B. 24h after experiment;

C. $48 \mathrm{~h}$ after experiment; D. $72 \mathrm{~h}$ after experiment

As Figure 1 above, change of $2 \mathrm{mg} / \mathrm{ml}$ PLGA/(CS/HA)4/CD34/HA multilayers after $24 \mathrm{~h}$ is not very obvious; fluorescence area gradually becomes smaller after $48 \mathrm{~h}$ and $72 \mathrm{~h}$, but there is still a considerable remaining area. The fast-coated stent endothelialization can complete endothelial reconstruction within 72 hours, so the results of the three groups test show that, PLGA/ (HA/CS) n/CD34 coated stent has good anti erosion ability, and is able to meet the requirement of vascular endothelial surface reconstruction after implantation.

\section{B. Hemocompatibility of Multilayers}

Conduct Scanning electron microscopy of platelets incubated on the surface of $1 \mathrm{mg} / \mathrm{ml}$, $2 \mathrm{mg} / \mathrm{ml} \quad \mathrm{PLGA} /(\mathrm{HA} / \mathrm{CS}) 2 / \mathrm{HA}$ and PLGA/(HA/CS)4/HA, and $5 \mathrm{mg} / \mathrm{ml}$ PLGA/(HA/CS)2/HA, by sheet stainless steel as matrix, and the scan results are shown as Figure 2. A, B, C and D are scanning electron microscope images of low-concentrations platelet adhesion of the coating surface, where we can see that the coating surfaces are smooth, adhered by more blood platelet, and pseudopod formation is also more evident. The results show: the layer number and concentration of multilayer prepared by low-concentration HA and CS solution almost has no effect on platelet adhesion and pseudopodia formation, and compared with high-concentration coating, it can more effectively reduce adhesion to platelet and formation of pseudopodia. In addition, it has good biocompatibility and anticoagulant properties. 

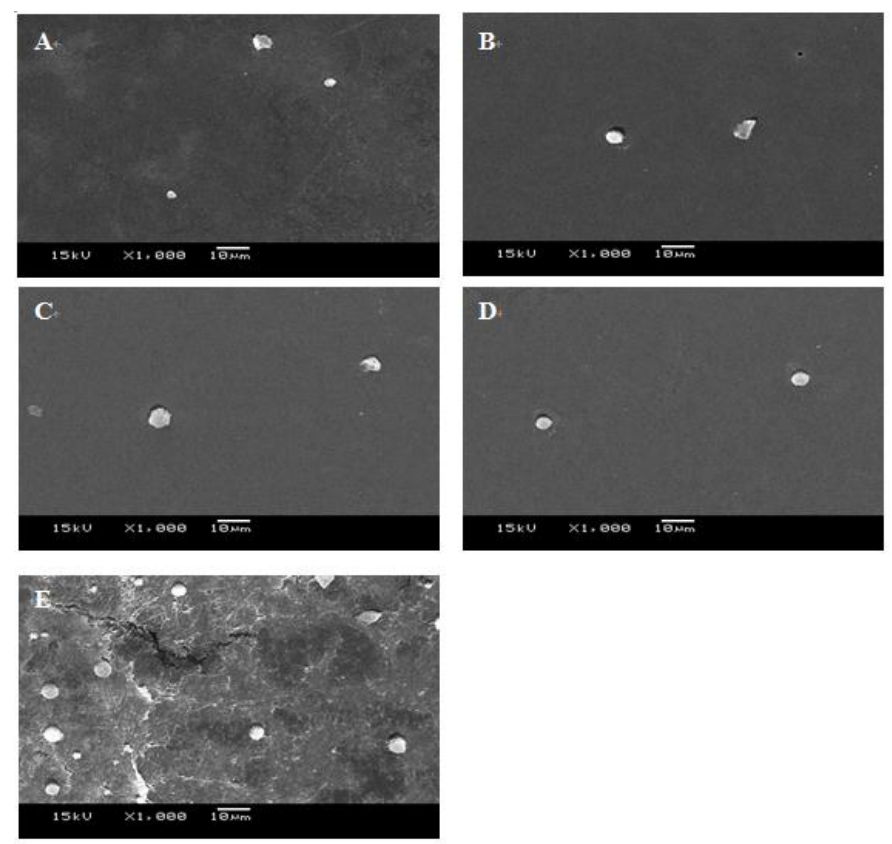

Figure 2. SEM images of platelet adhesion on coating. A. the Concentration of PLGA/ PEI+/ (HA-/Cs+) 2/ $\mathrm{HA}$ - is $1 \mathrm{mg} / \mathrm{ml}$;

B. the Concentration of PLGA/ PEI+/（HA-/Cs+） 4/ $\mathrm{HA}$ - is $1 \mathrm{mg} / \mathrm{ml}$;

C. the Concentration of PLGA/ PEI+/（HA-/Cs+）2/ $\mathrm{HA}$ - is $2 \mathrm{mg} / \mathrm{ml}$;

D. the Concentration of PLGA/ PEI+/（HA-/Cs+） 4/ $\mathrm{HA}$ - is $2 \mathrm{mg} / \mathrm{ml}$;

E. the Concentration of PLGA/ PEI+/（HA-/Cs+）2/ $\mathrm{HA}$ - is $5 \mathrm{mg} / \mathrm{ml}$

\section{Conclusions}

In this paper, we adopt electrostatic self-assembly method to fabricate PLGA/(HA/CS)n/CD34 bioactive multiplayer film on stainless steel stent, and characterize the antibody in multilayers with fluorescence labeling. In erosion experiments in vitro, we place the stent scribbled with bioactive multiplayer in the flow simulated body fluid respectively for $24 \mathrm{~h}, 48 \mathrm{~h}$ and $72 \mathrm{~h}$, and then observe the fluorescence microscopy picture of antibody coating and Rhodamine-labeled goat anti-mouse IgG antibody combined. The results show that there is still a lot of antibody remaining on the surface of biological coating stent, so it has good erosion resistant performance. That multiplayer has less platelet adhesion and pseudopod formation, so it has good biocompatibility, providing theoretical basis for experiment after implantation of vascular.

\section{REFERENCE:}

[1] Ferns G A, Avades T Y. The Mechanisms of Coronary Rest - enosis : Insights from Experimental Models[J j. Int J ExpPathol, 2000, 81(2): $63-88$.

[2] Stone GW , EllisSG , CoxDA , eta1. Apolymer-based, paelitaxeleluting stent in patients with coronary artery disease. $\mathrm{N}$ Engl $\mathrm{J}$ Med, 2004, 350(3): 221-31.

[3] Holmes DR Jr.State of the art in coronary intervention[J].Am Cardiol,2003,91(3A):50A-53A.

[4] Fattori R,Piva T.Drug-eluting stents in vascular intervention[J]. Lancet, 2003, 361 (9353): 247-249.

[5] Alkrad Jamal Alyoussef, Mrestani Yahya, Neubert Reinhard HH. The release profiles of intact and enzymatically digested hyaluronic acid from semisolid form ulations using multi-layer membrane system[J]. European Journal of Pharmaceutics and Biopharmaceuti-cs, 2003, 56: $37-41$. 